\title{
Caracterização climática de Cachoeira do Sul, RS quanto à precipitação pluvial
}

Valeria Pohlmann

Marcondes Lazzari
Universidade Estadual do Rio Grande do Sul (UERGS). Unidade Universitária em Cachoeira do Sul.

E-mail: valeriapohlmann@hotmail.com marcondes-lazzari@uergs.edu.br

Recebido em: 20 set. 2017. Revisado em: 20 dez. 2017. Aceito: 22 dez. 2017.

DOI: http://dx.doi.org/10.21674/2448-0479.41.42-60

\section{Resumo}

Entre as importâncias da caracterização climática para diferentes setores da economia, podemos destacar a agricultura, uma vez que estas informações podem subsidiar diversas tomadas de decisões. A precipitação pluvial apresenta grande variabilidade espaçotemporal e constitui uma das principais fontes de perdas na produtividade agrícola. Desta forma, este trabalho teve por objetivo caracterizar a precipitação pluvial (PP) de Cachoeira do Sul. Utilizou-se dados dos anos de 2007 a 2016 do Instituto Nacional de Meteorologia (INMET) da estação meteorológica A813 (lat: -2952'; long: 52²3'; alt: 107m) e dos anos de 1982 a 2007 da Cooperativa Agrícola Cachoeirense (CORISCAL) (lat: $-30^{\circ} 2^{\prime}$; long: $-52^{\circ} 53^{\prime}$; alt: $64 \mathrm{~m}$ ). Os dados foram obtidos a $1,5 \mathrm{~m}$ e analisados por meio do Excel. Os resultados foram comparados à Normal Climatológica (NC) 1961/1990. Analisando dados do INMET, verificou-se que a PP média anual foi de $1646,7 \mathrm{~mm}( \pm 263,5 \mathrm{~mm})$, distribuídas em 104 dias $( \pm 10,4$ dias) com PP $\geq 1 \mathrm{~mm}$. Dados da CORISCAL indicam PP mé- 
dia de $1738,9 \mathrm{~mm}( \pm 362,6 \mathrm{~mm})$ em 68 dias $( \pm 10,0$ dias $)$. Percebe-se que a precipitação pluvial média anual e mensal é semelhante à Normal Climatológica analisada. O conhecimento sobre estas informações possibilita melhores escolhas sobre diversos fatores, como o manejo do solo e seleção de espécies mais adaptadas à região.

Palavras-chave: Normal climatológica. Tomadas de decisões. Agricultura.

\section{Abstract \\ Climatic characterization of Cachoeira do Sul, RS regarding rainfall}

Among the importance of the climatic characterization for different sectors of the economy, we can highlight agriculture, since this information can subsidize several decisions. Rainfall presents great spatio-temporal variability and is one of the main sources of losses in agricultural productivity. The aim of this work was to characterize the rainfall (PP) of Cachoeira do Sul. Data from the years 2007 to 2016 of the National Institute of Meteorology (INMET) of meteorological station A813 (lat: $29^{\circ} 52$ ', long: $-52^{\circ} 23^{\prime}$, alt: $107 \mathrm{~m}$ ) and from the years 1982 to 2007 of the Cooperativa Agrícola Cachoeirense (CORISCAL) (lat: $-30^{\circ} 2$ '; long: $-52^{\circ} 53^{\prime}$; alt: $64 \mathrm{~m}$ ). Data were obtained at $1.5 \mathrm{~m}$ and analyzed using Excel. The results were compared to Normal Climatology (NC) 1961/1990. Analyzing data from INMET, it was verified that the annual mean PP was $1646.7 \mathrm{~mm}( \pm 263.5 \mathrm{~mm})$, distributed in 104 days ( \pm 10.4 days) with PP $\geq 1 \mathrm{~mm}$. Data from CORISCAL indicate mean PP of $1738.9 \mathrm{~mm}( \pm 362.6 \mathrm{~mm})$ in 68 days $( \pm$ 10.0 days). It is noticed that the average annual rainfall and monthly rainfall is similar to the Normal Climatological analyzed. Knowledge 
about this information makes it possible to make better choices about several factors, such as soil management and selection of species more adapted to the region.

Keywords: Normal climatological. Decision-making. Agriculture.

\section{Introdução}

O clima de qualquer região, localizado nas diversas latitudes do globo, não possui as mesmas características em cada ano, pois ocorrem variações ao longo destes (SORIANO, 1997). Deste modo, a Organização Meteorológica Mundial (OMM) estabelece que para estudos comparativos do clima, sejam calculadas médias climatológicas para períodos de trinta anos de observação. Entretanto, períodos mais curtos, desde que realizados para anos sucessivos de no mínimo dez anos, denominada normal provisória, prestam-se para avaliar o comportamento do clima (OMM, 1989).

Os elementos meteorológicos podem variar ao longo da safra, ocasionando incertezas na produção agrícola. A precipitação pluvial apresenta grande variabilidade espaço-temporal (AYOADE, 2004) e constitui uma das principais fontes de perdas na produtividade agrícola (MONTEIRO, 1976). Nas zonas rurais, as irregularidades no regime pluviométrico resultam na perda parcial ou total das safras, que comprometem o mercado, o desemprego e a segurança alimentar (ELY; ALMEIDA; SANT'ANNA NETO, 2003).

O fenômeno El Niño-Oscilação do Sul (ENOS), por sua vez, contribui para a variabilidade da precipitação pluvial no Brasil, possuindo duas modalidades, o El Niño que demonstra o aquecimento das temperaturas do oceano Pacífico Tropical e a La Niña que representa o resfriamento do mesmo. Ambos estão associados 
aos campos de pressão representados pelo Índice de Oscilação do Sul (IOS) que alteram o padrão de circulação da atmosfera, afetando várias regiões do mundo de maneira distinta. No Sul do Brasil, em decocorrência do EI Niño, há elevada precipitação pluvial e, em anos com La Niña, estiagens. A influência dos fenômenos ocorre durante todo o período de atuação, havendo duas épocas do ano que são mais afetadas, que seriam outubro, novembro e dezembro no ano inicial do evento, e abril, maio e junho no ano seguinte ao início do evento (CUNHA, 2003). Essa anomalia climática afeta o comportamento hídrico das regiões, influenciando a produção agrícola. Fontana; Berlato (1996) no Rio Grande do Sul realizaram estudos que comprovam que em anos de El Niño há precipitação pluvial acima da Normal e que em anos com La Niña, abaixo da Normal.Tal fato foi verificado em todos os meses, principalmente na primavera do ano de início do fenômeno e no final do outono do ano seguinte.

O Índice de Oscilação do Sul (IOS) é o mais comumente usado para medir as intensidades do fenômeno ENOS. Cunha (2003, p.2) relata que o IOS:

\footnotetext{
Reflete as diferença de pressão atmosférica entre duas estações-chave para o fenômeno (Darwin e Taiti) e a temperatura da superfície do mar (TSM), em uma região chamada de Niño $3\left(5^{\circ} \mathrm{N}-5^{\circ} \mathrm{S}\right.$ e $\left.90^{\circ}-150^{\circ} \mathrm{W}\right)$. O IOS mede a intensidade da Oscilação do Sul (componente atmosférico) e a TSM da região Niño 3 mede a intensidade do EL Niño (componente oceânico).
}

Na região tropical, a precipitação pluvial, sob um olhar hidrológico, é a de maior interesse por predominar e ser quantitativamente superior em eficiência (MARIANO; SANTOS; SCOPEL, 2006). Sua importância reside na recarga dos mananciais hídricos, essenciais para o consumo humano, animal, agrícola e industrial. A quantidade e a distribuição da precipitação pluvial que ocorre anualmente 
em uma região determinam o tipo de vegetação natural e também o modo de exploração agrícola predominante (BURIOL et al., 2007). O clima é observado para auxiliar no gerenciamento das atividades agrícolas. Essa gestão inclui o planejamento temporal e manejo de operações como semeadura, colheita, plantio, aplicação de defensivos agrícolas, preparo do solo e logística no transporte e armazenamento dos produtos (DAS et al., 2010). O uso de informações climáticas é essencial para apoiar as tomadas de decisões em nível da propriedade, sendo que a bases de dados climáticos são fundamentais para aumentar o conhecimento local com intuito de prover soluções para problemas enfrentados na agricultura (BAMBINI, 2011).

Para a agricultura, a precipitação pluvial é fundamental para seu bom funcionamento, principalmente para culturas não irrigadas. Entretanto, para as irrigadas, o estudo na captação e condução das águas também é dependente destas informações, podendo embasar melhores escolhas quanto ao planejamento, uso e manejo mais adequado do solo, irrigação, colheita e relação entre plantas, microorganismos e insetos (GHINI; HAMADA; BETTIOL, 2011).

A produtividade agrícola no Brasil depende do clima e sua variabilidade, sendo responsável pela alternância das produções agrícolas anuais (INTERGOVERNMENTAL PANEL ON CLIMATE CHANGE, 2001). Vários estudos demonstram as consequências causadas pela oscilação da precipitação pluvial em vários cultivos agrícolas. As irregularidades na precipitação pluvial em 1967/1968, para a cultura de arroz em São Paulo, provocaram queda na safra (GUADARRAMA, 1971). Em outro estudo, Sant' Anna Neto (1998) relatou que a variabilidade e irregularidade da precipitação pluvial e as intempéries climáticas interferem na fenologia das plantas, diminuindo a eficiência da produção e comprometendo os calendários 
agrícolas. Sobre o cultivo da soja nas regiões de Cascavel e Toledo no estado do Paraná, Almeida (2000) constatou concordância espacial entre regiões que obtiveram melhores rendimentos e áreas onde a oferta da precipitação foi mais elevada. Para o município de Coromandel em Minas Gerais, Santos; Ribeiro (2002) concluíram que a precipitação pluvial é fator influenciador na produtividade agrícola, sendo períodos com deficiência hídrica nas fases reprodutivas, principalmente janeiro e fevereiro e excesso hídrico na colheita, as condições que causam maiores prejuízos. No Rio Grande do Sul, Berlato; Cordeiro (2005, p.48) destacam que "a maior variabilidade dos rendimentos coincide com a maior variabilidade da precipitação pluvial". Silva; Prela-Pantano; Neto (2008) concluíram que a variabilidade interanual da precipitação pluvial no Vale do Médio Paranapanema influencia a produtividade agrícola, sendo as culturas anuais como milho e soja, que são cultivadas em pequenas e médias propriedades, as que mais sofrem. Silva; Souza (2012) elaboraram uma análise sobre a influência do regime pluvial na primavera-verão de 2005/2006, relacionando estas informações com dados de produtividade para os 10 maiores municípios produtores de soja em Tocantins e concluíram que os meses de outubro e janeiro foram secos e o mês de março chuvoso, os quais influenciaram na perda de produtividade de soja em cinco dos maiores municípios produtores.

O município de Cachoeira do Sul caracteriza-se por possuir economia principal oriunda do setor agropecuário. $\mathrm{O}$ estudo referente à caracterização climática da região é de suma importância para subsidiar uma produção agropecuária mais sustentável, fornecendo suporte ao planejamento agrícola na região. $\mathrm{O}$ objetivo deste trabaIho foi caracterizar a precipitação pluvial $(\mathrm{mm})$ de Cachoeira do Sul. 


\section{Material e Métodos}

O município de Cachoeira do Sul está localizado na depressão central do Rio Grande do Sul, na latitude -30¹4'09" e longitude $52^{\circ} 58^{\prime} 43^{\prime \prime}$, com altitude média de $64 \mathrm{~m}$. O clima, segundo classificação de Köppen; Geiger (1928), é subtropical (CFa) com clima úmido e verão quente.

Para a realização do trabalho, utilizou-se dados diários de precipitação pluvial $(\mathrm{mm})$ dos anos de 1982 a 2007, totalizando vinte e seis anos, obtidos na Cooperativa Agrícola Cachoeirense (CORISCAL), com latitude de - $30^{\circ} 02^{\prime} 32^{\prime \prime}$, longitude de $-52^{\circ} 53^{\prime} 09^{\prime \prime}$ e altitude de $64 \mathrm{~m}$ em Cachoeira do Sul. Da estação meteorológica automática A813 do Instituto Nacional de Meteorologia (INMET), da marca Vaisala, modelo MAWS 301, localizada em Rio Pardo, distante do centro de Cachoeira do Sul em 51,8km, com latitude de $29^{\circ} 52^{\prime} 19^{\prime \prime}$, longitude de $-52^{\circ} 22^{\prime} 55^{\prime \prime}$ e altitude de $107 \mathrm{~m}$ utilizou-se dados horários de precipitação pluvial $(\mathrm{mm})$ dos anos de 2007 a 2016, totalizando dez anos. Os dados foram analisados através do programa Excel 2007.

Foram determinadas a precipitação pluvial acumulada média e o número de dias com precipitação pluvial $\geq 1 \mathrm{~mm}$ médios mensais, anuais e sazonais. Foram realizadas observações nas duas épocas do ano que são mais afetadas pelos fenômenos ENOS, que são caracterizados por anomalias positivas (El Niño) ou negativas (La Niña), em outubro, novembro e dezembro no ano inicial do evento e abril, maio e junho no ano seguinte ao início do evento (CUNHA, 2003). Foi analisada a distribuição noturna e diurna da precipitação pluvial, sendo que considerou-se dia das $07 \mathrm{~h}$ às $18 \mathrm{~h}$ e noite das $19 \mathrm{~h}$ às $6 \mathrm{~h}$, totalizando doze horas em cada turno. Foi calculado o desvio padrão para as médias analisadas. Sobre os dados obtidos, reali- 
zou-se uma comparação com os dados existentes da Normal Climatológica de 1961 a 1990 elaboradas pelo Instituto Nacional de Meteorologia.

\section{Resultados e Discussão}

A Normal Climatológica possui em média $1477,1 \mathrm{~mm}$ anuais, podendo-se observar que embora as médias estudadas estejam com valor superior à normal, estão dentro do desvio padrão analisado com relação à Normal, com média do INMET de 1646,7mm $( \pm 263,5 \mathrm{~mm})$ e da CORISCAL com $1738,9 \mathrm{~mm}( \pm 362,6 \mathrm{~mm})$ (Tabela 1). De acordo com Moreno (1961), no norte e centro do Rio Grande do Sul, os valores médios apresentam aumento gradativo, apresentando nas áreas mais elevadas acúmulos superiores a $1600 \mathrm{~mm}$. Para a estação experimental da Universidade Federal do Rio Grande do Sul, localizada em Eldorado do Sul, o acúmulo médio foi de 1440mm (BERGAMASCHI et al., 2003). De acordo com Tubelis; Nascimento (1992) o Estado possui média anual de $1555 \mathrm{~mm}$. O número de dias com precipitação pluvial $\geq 1 \mathrm{~mm}$ médio anual de 2007 a 2016 apresentou aumento em comparação com os anos antecessores (Tabela 2).

A precipitação pluvial total média da região é superior às médias do Litoral Sul e Campanha, inferior às da Serra do Nordeste, Planalto, Missões e Alto Vale do Uruguai e se assemelha ao restante da Depressão Central, Litoral Norte, Baixo Vale do Uruguai e Serra do Sudeste (MORENO, 1961; BEIRSDORF; MOTA, 1973; BURIOL; ESTEFANEL; FERREIRA, 1977). De acordo com dados do IN$\mathrm{MET}$, o mês de maior acúmulo de precipitação pluvial é setembro e os de menor são março e abril. Entretanto, de acordo com os desvi- 
os padrões observados, o mês com maior variabilidade de acúmulo é novembro e o menor, abril. Segundo dados da CORISCAL, a maior média mensal acumulada e variabilidade observada ocorreram no mês de outubro e as menores em março (Tabela 1). Maluf; Gessinger; Cunha (1981) mencionam que outubro é o mês com maior valor médio de precipitação pluvial e novembro, o de menor. Em outro estudo, Tubelis; Nascimento (1992) apontam que o mês com menor precipitação média é novembro e o de maior é outubro. A precipitação pluvial é um dos elementos meteorológicos mais variáveis (BERGAMASCHI et al., 2003).

A precipitação pluvial anual é bem distribuída em todos os meses do ano e não há estação seca definida. Deste modo, permite caracterizar o clima da região, com relação à precipitação pluvial, como Cfa, pela classificação de Köppen; Geiger (1928), ou seja, subtropical úmido.

Com o aquecimento global, a frequência e a intensidade das precipitações pluviais apresentam aumento. Porém, com a degradação dos solos, aliados às altas intensidades pluviométricas, os solos não possuem a capacidade de absorver e reter totalmente a quantidade de água disponível em curto período de tempo, ocasionando escoamento superficial, erosão, assoreamento dos rios e alagamentos. Com base em simulações de modelos globais para uma ampla gama de cenários, há tendência de aumento da precipitação pluvial durante o século 21 , sendo que na segunda metade do século é provável que em baixas latitudes ocorram aumentos e diminuições das precipitações em diferentes regiões (INTERGOVERNMENTAL PANEL ON CLIMATE CHANGE, 2001). Do mesmo modo, Blain et al. (2009) concluíram que, para Pelotas/RS, existe a tendência de elevação no regime de precipitação pluvial ocorrida entre os anos de 1948-1976 e 1977-2005. Groisman et al. (2005) identificaram ten- 
dências positivas de aumentos da precipitação pluvial e de seus extremos na região subtropical, no Sul e no Nordeste do Brasil. Analisando a tendência temporal da precipitação pluvial no Rio Grande do Sul, de 1950 a 2002, Berlato et al. (2007) concluíram aumento significativo da precipitação no total anual e no outono. Marengo (2007) relata que o aumento da precipitação pluvial pode também ser observado nos registros hidrológicos, onde as vazões do Rio Jacuí, em Espumoso e em Passo Bela Vista, apresentam tendências positivas. Porém, os dados analisados encontram-se dentro da Normal comparada, não apresentando acréscimo ou decréscimo na média acumulada anual.

Tabela 1. Precipitação pluvial acumulada $(\mathrm{mm})$ e seus respectivos desvios padrões (s) das fontes de dados do INMET, CORICAL e Normal Climatológica. Cachoeira do Sul, RS.

\begin{tabular}{cccccc}
\hline & INMET & s & $\begin{array}{c}\text { CORICAL } \\
\mathbf{1 9 8 2 - 2 0 0 7}\end{array}$ & s & $\begin{array}{c}\text { NORMAL } \\
\text { CLIMATOLÓGICA } \\
\mathbf{1 9 6 1 - 9 0}\end{array}$ \\
\hline Jan & 114,0 & $( \pm 61,2)$ & 120,9 & $( \pm 73,6)$ & 127,6 \\
Fev & 143,5 & $( \pm 36,4)$ & 146,7 & $( \pm 76,5)$ & 111,2 \\
Mar & 97,2 & $( \pm 53,6)$ & 114,0 & $( \pm 67,1)$ & 121,1 \\
Abr & 97,2 & $( \pm 51,1)$ & 169,0 & $( \pm 95,4)$ & 83,6 \\
Mai & 103,3 & $( \pm 46,0)$ & 144,0 & $( \pm 101,9)$ & 93,3 \\
Jun & 125,8 & $( \pm 56,4)$ & 165,5 & $( \pm 96,6)$ & 138,6 \\
Jul & 173,1 & $( \pm 65,5)$ & 174,2 & $( \pm 97,7)$ & 145,3 \\
Ago & 141,6 & $( \pm 75,2)$ & 122,4 & $( \pm 76,1)$ & 157,4 \\
Set & 187,4 & $( \pm 66,0)$ & 122,4 & $( \pm 80,2)$ & 148,4 \\
Out & 185,1 & $( \pm 98,4)$ & 176,2 & $( \pm 106,2)$ & 124,9 \\
Nov & 156,5 & $( \pm 157,0)$ & 129,5 & $( \pm 78,4)$ & 112,1 \\
Dez & 138,0 & $( \pm 66,9)$ & 115,1 & $( \pm 74,6)$ & 113,7 \\
\hline Anual & $\mathbf{1 6 4 6 , 7}$ & $\mathbf{( \pm 2 6 3 , 5 )}$ & $\mathbf{1 7 3 8 , 9}$ & $\mathbf{( \pm 3 6 2 , 6 )}$ & $\mathbf{1 4 7 7 , 1}$ \\
\hline
\end{tabular}


Tabela 2. Dias com precipitação pluvial $\geq 1 \mathrm{~mm}$ e seus respectivos desvios padrões (s) das fontes de dados do INMET, CORICAL e Normal Climatológica. Cachoeira do sul, RS.

\begin{tabular}{cccccc}
\hline & $\begin{array}{c}\text { INMET } \\
\mathbf{2 0 0 7 - 1 6}\end{array}$ & s & $\begin{array}{c}\text { CORISCAL } \\
\mathbf{1 9 8 2 - 2 0 0 7}\end{array}$ & s & $\begin{array}{c}\text { NORMAL } \\
\text { CLIMATOLÓGICA } \\
\mathbf{1 9 6 1 - 9 0}\end{array}$ \\
\hline Jan & 8,0 & $( \pm 3,0)$ & 6,0 & $( \pm 3,0)$ & 9,0 \\
Fev & 10,0 & $( \pm 1,7)$ & 6,0 & $( \pm 3,0)$ & 7,0 \\
Mar & 8,0 & $( \pm 2,2)$ & 5,0 & $( \pm 2,2)$ & 8,0 \\
Abr & 7,0 & $( \pm 2,8)$ & 6,0 & $( \pm 2,5)$ & 6,0 \\
Mai & 7,0 & $( \pm 2,2)$ & 5,0 & $( \pm 3,0)$ & 6,0 \\
Jun & 9,0 & $( \pm 2,6)$ & 6,0 & $( \pm 2,5)$ & 8,0 \\
Jul & 9,0 & $( \pm 2,7)$ & 6,0 & $( \pm 3,2)$ & 9,0 \\
Ago & 9,0 & $( \pm 3,6)$ & 5,0 & $( \pm 2,8)$ & 9,0 \\
Set & 10,0 & $( \pm 2,4)$ & 6,0 & $( \pm 2,2)$ & 9,0 \\
Out & 9,0 & $( \pm 3,1)$ & 7,0 & $( \pm 2,5)$ & 8,0 \\
Nov & 7,0 & $( \pm 3,6)$ & 5,0 & $( \pm 2,7)$ & 7,0 \\
Dez & 9,0 & $( \pm 3,9)$ & 5,0 & $( \pm 1,7)$ & 7,0 \\
\hline Anual & $\mathbf{1 0 4 , 0}$ & $\mathbf{( \pm 1 0 , 4 )}$ & $\mathbf{6 8 , 0}$ & $\mathbf{( \pm 1 0 , 0 )}$ & $\mathbf{9 3 , 0}$ \\
\hline
\end{tabular}

Torna-se complexo explicar a variação espacial da precipitação pluvial, pois entre tantos elementos climatológicos, este é um dos mais variáveis nas tantas distintas localidades, por isso a importância do estudo de seu comportamento. Sobre a confiabilidade das variações, Ayoade (2004) menciona que quanto menor a variabilidade, maior é a confiabilidade dos dados sobre a precipitação média em um determinado lugar, enquanto a alta variabilidade implica amplas flutuações em torno do valor médio.

A pequena variabilidade interanual da precipitação pluvial média sobre o Rio Grande do Sul não permite caracterizar claramente estações chuvosas e secas ao longo do ano. Entretanto, a 
variabilidade interanual da precipitação pluvial é apontada como a principal causa da variação dos rendimentos agrícolas no Rio Grande do Sul (BERLATO; FONTANA, 1999).

A distribuição sazonal da precipitação pluvial é tão importante quanto o volume total. Percebe-se que a estação com maior número de dias com precipitação pluvial em ambas as fontes é o inverno (Tabela 3). O número de dias com precipitação pluvial foi semelhante nas quatro estações do ano, resultados similares aos encontrados para o município de Santa Maria (SILVA et al., 2007) e para o Rio Grande do Sul (FONTANA; ALMEIDA, 2002). Dados do INMET indicam que a estação com maior volume pluviométrico médio é o inverno, com $478,9 \mathrm{~mm}( \pm 94,2 \mathrm{~mm})$, representando $29 \%$ da distribuição sazonal. Dados da CORISCAL mostram o outono com $478,5 \mathrm{~mm}( \pm 199,1 \mathrm{~mm})$, representando $28 \%$ da distribuição sazonal da precipitação pluvial. Considerando a variabilidade sazonal, a primavera, de acordo com dados do INMET, pode ser a estação com maior volume, o que corrobora com Maluf et al. (1981), que apontam a primavera como sendo a estação com maior acúmulo, seguida por outono, inverno e verão. Quanto à sua distribuição, os dados destoam de Berlato (1992), que menciona que a distribuição sazonal da precipitação pluvial no Rio Grande do Sul é de $26 \%$ na primavera, $24 \%$ no verão, $25 \%$ no outono e $25 \%$ no inverno. 
Tabela 3. Precipitação pluvial (PP) $(\mathrm{mm})$ e dias com precipitação pluvial $\geq 1 \mathrm{~mm}$ e seus respectivos desvios padrões (s) para as estações do ano das fontes de dados do INMET e CORICAL. Cachoeira do Sul, RS.

\begin{tabular}{lccccccccc}
\hline & \multicolumn{3}{c}{ INMET 2007-2016 } & \multicolumn{3}{c}{ CORISCAL 1982-2007 } \\
\hline & PP & s & $\begin{array}{c}\text { Dias } \\
\text { PP } \\
\geq 1 \mathrm{~mm}\end{array}$ & s & PP & s & $\begin{array}{c}\text { Dias } \\
\text { PP } \\
\geq 1 \mathbf{m m}\end{array}$ & s \\
\hline Prim & 461,2 & $( \pm 196,6)$ & 25 & $( \pm 5,2)$ & 417,0 & $( \pm 176,5)$ & 16 & $( \pm 4,2)$ \\
Ver & 399,0 & $( \pm 107,2)$ & 27 & $( \pm 4,7)$ & 374,0 & $( \pm 116,6)$ & 17 & $( \pm 4,8)$ \\
Out 288,7 & $( \pm 99,4)$ & 22 & $( \pm 6,2)$ & 479,0 & $( \pm 199,1)$ & 17 & $( \pm 5,1)$ \\
Inv & 478,9 & $( \pm 94,2)$ & 28 & $( \pm 5,2)$ & 470,0 & $( \pm 160,4)$ & 18 & $( \pm 5,3)$ \\
\hline
\end{tabular}

O conhecimento sobre a distribuição da precipitação pluvial noturna e diurna é importante para subsidiar as escolhas sobre o manejo nas propriedades. Segundo dados do INMET, o dia possui em média $788,4 \mathrm{~mm}( \pm 155,6 \mathrm{~mm})$ anuais e a noite, $852,4 \mathrm{~mm}$ $( \pm 72,9 \mathrm{~mm})$, sendo a noite o período que mais chove, totalizando $52 \%$. O mês mais chuvoso durante o dia e a noite é setembro com $100,2 \mathrm{~mm}( \pm 55,7 \mathrm{~mm})$ e $101,6 \mathrm{~mm}( \pm 70,6 \mathrm{~mm})$, respectivamente.

A boa distribuição da precipitação pluvial durante os meses do ano faz com que os períodos de temperatura mais amena, no inverno, favoreçam a ocorrência de patógenos que podem prejudicar a produção de hortaliças folhosas produzidas fora de ambientes protegidos. As plantas permanecem por mais tempo com água líquida sobre seus tecidos, favorecendo a infecção de patógenos. $O$ mofo branco (Sclerotinia stem) em condições de campo necessita em torno de $39 \mathrm{~h}$ de molhamento foliar para iniciar a infecção, e em ambiente protegido, $54 \mathrm{~h}$, ambas a $20^{\circ} \mathrm{C}$ (BOLAND; HALL, 1988).

A primavera/verão apresenta bom volume de precipitações que são favoráveis às culturas anuais de grãos na região, como o 
milho, que necessita aproximadamente $650 \mathrm{~mm}$ para obter rendimento máximo (BERGAMASCHI et al., 2001), o arroz de terras altas, que possui demanda hídrica entre 450 a $700 \mathrm{~mm}$ durante todo ciclo (RODRIGUES; SORATTO; ARF, 2004), a soja, que necessita entre 450 a $850 \mathrm{~mm}$ para obtenção de produtividade considerável (FRANKE, 2000) e o feijão, que consome cerca de $300 \mathrm{~mm}$ de água em todo o ciclo (BALARDIN et al., 2000).

Os fenômenos ENOS apresentam grande influência no comportamento hídrico da região Sul do país. O ano de 2009/2010 possuiu o fenômeno El Niño de intensidade moderada, apresentando $1133 \mathrm{~mm}$ nos meses outubro, novembro e dezembro de 2009 e abril, maio e junho de 2010. Do mesmo modo, o ciclo 2012/2013 possuiu 1348,2mm. Em contrapartida, 2011/2012 apresentou $475,4 \mathrm{~mm}$, sendo considerada La Niña de baixa intensidade. Segundo a Normal Climatológica analisada, a média de precipitação pluvial acumulada para estes meses seria de $728 \mathrm{~mm}$, comprovando que em ano com El Niño a precipitação é superior à Normal e em anos de La Niña é inferior.

\section{Conclusão}

A precipitação pluvial anual e mensal são semelhantes à Normal Climatológica analisada. Em ano com El Niño, a precipitação pluvial é superior à Normal e em anos de La Niña é inferior. A posse destas informações facilita as tomadas de decisões no meio agrícola e urbano e em várias áreas do conhecimento.

\section{Referências}


ALMEIDA, I.R. de. Variabilidade pluviométrica interanual e produção de soja no Estado do Paraná. 2000. 200f. Dissertação (Mestrado em Geografia) - Faculdade de Ciências e Tecnologia, Universidade Estadual Paulista, Presidente Prudente, 2000.

AYOADE, J.O. Introdução à climatologia para os trópicos. Tradução Maria Juraci Zani dos Santos. 10 ed. Rio de Janeiro: Bertrand Brasil, 2004.

BALARDIN, R.S. et al. Feijão: recomendações técnicas para cultivo no Rio Grande do Sul/ Comissão Estadual de Pesquisa de Feijão. Biblioteca Setorial do Centro de Ciências Rurais/UFSM 2000.

BAMBINI, M.D. Inovação tecnológica e organizacional em agrometeorologia: estudo da dinâmica da rede mobilizada pelo sistema Agritempo. 2011. Dissertação (Mestrado em Política Científica e Tecnológica) - Universidade Estadual de Campinas, 2011.

BEIRSDORF, M.I.C.; MOTA, F.S. da. Mapa pluviométrico anual do Rio Grande do Sul e Santa Catarina. Ciência e Cultura, São Paulo, v. 25, n. 15 , p. 457-60, 1973.

BERGAMASCHI, $\mathrm{H}$ et al. Clima da Estação Experimental da UFRGS (e região de abrangência). Porto Alegre, 2003.

BERGAMASCHI, $\mathrm{H}$. et al. Estimating maize water requirements using agrometeorological data. Revista Argentina de Agrometeorologia, v.1, p.23-27.7 2001.

BERLATO, M. A. As condições de precipitação pluvial no Estado do Rio Grande do Sul e os impactos da estiagem na produção agrícola. In: Bergamaschi, H. (coord.) Agrometeorologia aplicada à irrigação. Porto Alegre: UFRGS, 1992. p.11-23.

BERLATO, M.A.; CORDEIRO, A.P.A. Variabilidade Climática e Agricultura do Rio Grande do Sul. In: FEDERACITE "As Estiagens e as Perdas na Agricultura: Fenômeno Natural ou Imprevidência"? Esteio. Anais... EXPOINTER, 2005. 
BERLATO, M.A.; FONTANA, D.S. Variabilidade interanual da precipitação pluvial e rendimento da soja no Estado do Rio Grande do Sul. Revista Brasileira de Agrometeorologia, Santa Maria, v. 7, n. 1. p. 119-125. 1999.

BERLATO, M.A. et al. Tendência observada da precipitação pluvial anual e estacional do Estado do Rio Grande do Sul e relação com a temperatura da superfície do mar do Oceano Pacífico. In: CONGRESSO BRASILEIRO DE AGROMETEOROLOGIA. Anais... Campinas: SBA, 2007.

BLAIN, G.C. et al. Variabilidade amostral das séries mensais de precipitação pluvial em duas regiões do brasil: Pelotas-RS e Campinas-SP. Revista Brasileira de Meteorologia, São José dos Campos, v. 24, n. 1, p. 1-11. 2009.

BOLAND, G.J.; HALL, R. Epidemiology of Sclerotinia stem rot of soybean in Ontario. Phytopathology, v. 78, p. 1241-1245. 1988.

BURIOL, G.A. et al. Clima e vegetação natural do Estado do Rio Grande do Sul segundo o diagrama climático de Walter e Lieth. Ciência Florestal, Santa Maria, v. 17, n. 2, p. 91-100. 2007.

BURIOL, G.A.; ESTEFANEL, V.; FERREIRA, M. Cartas mensais e anual de chuvas do Estado do Rio Grande do Sul. Revista Centro Ciências Rurais, Santa Maria, v. 7, n.1, p. 55-82. 1977.

CUNHA, G.R. La Niña e a agricultura no sul do Brasil. 2003. Disponível em: <http://www.cnpt.embrapa.br/pesquisa/agromet/agromet/elninolanina /plantar.html>. Acesso em 10 jul 2015.

DAS, H.P. et al. Chapter 5 - Weather and Climate Forecasts for Agriculture. In: Guide to Agricultural Meteorological Practices, $p$ 5-1 a 5-57. 2010.

ELY, D.F.; ALMEIDA, I.R.; SANT'ANNA NETO, J.L. Implicações políticas e econômicas, variabilidade climática e o rendimento da 
cultura do milho no estado do Paraná. Revista do Departamento de Geociências, Londrina, v. 12, n. 1, p. 495-508. 2003.

FONTANA, D.C.; ALMEIDA, T.S. Climatologia do número de dias com precipitação pluvial no estado do Rio Grande do Sul. Revista Brasileira de Agrometeorologia, Santa Maria, v.10, n.2, p.341-349. 2002.

FONTANA, D.C., BERLATO, M.A. Influência do El Niño Oscilação Sul sobre a precipitação do Estado do Rio Grande do Sul. Revista Brasileira de Agrometeorologia. Santa Maria, v. 5, n. 1, p. 127132. 1996.

FRANKE, A.E. Necessidade de irrigação suplementar em soja nas condições edafoclimáticas do Planalto Médio e Missões, RS. Revista Pesquisa Agropecuária Brasileira, Brasília, v. 35 n.8. 2000.

GHINI, R.; HAMADA, E.; BETTIOL, W. Impactos das mudanças climáticas sobre doenças de importantes culturas no Brasil. Embrapa Meio Ambiente. 356p. 2011.

GROISMAN, P.Y. et al. Trends in intense precipitation in the climate record. Journal of Climate, n.18, p.1326-50. 2005.

GUADARRAMA, J.A.P. Ritmo pluvial e produção de arroz no Estado de São Paulo no ano agrícola de 1967-1968. São Paulo: Instituto da Geografia da USP, 1971.

INTERGOVERNMENTAL PANEL ON CLIMATE CHANGE (IPCC). HOUGHTON, J. T. et al. (eds.). The scientific basis. Cambridge: University Press, 2001. p. 892.

KÖPPEN, W.; GEIGER, R. Klimate der Erde. Gotha: Verlag Justus Perthes, 1928.

MALUF, J.R.T.; GESSINGER, G.I.; CUNHA, G.R. da. Agrometeorologia do Estado do Rio Grande do Sul. V - Precipitação pluviométrica, normal climatológica, 1931-60. In CONGRESSO 
BRASILEIRO DE AGROMETEOROLOGIA, 2. Anais... Pelotas, 1981. p.25-30.

MARENGO, J.A. Mudanças climáticas globais e seus efeitos sobre a biodiversidade: caracterização do clima atual e definição das alterações climáticas para o território brasileiro ao longo do século XXI. 2. ed. Brasília: Ministério do Meio Ambiente, v.1, p.214, 2007.

MARIANO, Z.F.; SANTOS, M.J.Z.; SCOPEL, I. A importância das chuvas para a produtividade da soja na microrregião do sudoeste de Goiás (GO). 2006. Disponível em: < http://www.rc.unesp.br/igce/geografia/pos/downloads/2006/a_import ancia.pdf>. Acesso em 05 nov. 2017.

MONTEIRO, C.A. de F. O clima e a organização do espaço de São Paulo: problemas e perspectivas. São Paulo: IGEOG/USP, 1976.

MORENO, J.A. Clima do Rio Grande do Sul. Porto Alegre: Secretaria da Agricultura, 1961.

ORGANIZAÇÃO METEOROLÓGICA MUNDIAL (OMM.). Calculation of monthly and annual $\mathbf{3 0}$ year standard normals. Geneva (WMO). Technical document, 1989. v. 341, n. 10.

RODRIGUES, R.A.F.; SORATTO, R.P.; ARF, O. Manejo de água em arroz de terras altas no sistema de plantio direto usando o tanque de Classe A. Engenharia Agrícola, Jabuticabal, v. 24, n. 3, p. 546-556. 2004.

SANT'ANNA NETO, J.L. Clima e a organização do espaço. Boletim de Geografia, Maringá, v. 16, n. 1, p. 119-131. 1998.

SANTOS, E.R. dos; RIBEIRO, A.G. Clima e agricultura no município de Caramandel-MG. In: SIMPÓSIO BRASILEIRO DE CLIMATOLOGIA GEOGRÁFICA, 5. 2002, Curitiba, Anais... Curitiba: UFPR, 2002. 
SILVA, A.A.F.; SOUZA, L.B. Análise da pluviosidade da primaveraverão 2005/2006 e influência na produtividade de soja no Tocantins. Revista GeoNorte, Manaus, v.2, p. 898-909. 2012.

SILVA, D.F.; PRELA-PANTANO, A.; NETO, J.L.S. Variabilidade da precipitação e produtividade agrícola na região do medio Paranapanema, SP. Revista Brasileira de Climatologia, Curitiba, v. 3/4, p. 101-116. 2008.

SILVA, J.C.; HELDWEIN, A.B.; MARTINS, F.B.; TRENTIN, G.; GRIM, E.L. Análise de distribuição de chuva para Santa Maria, RS. Revista Brasileira de Engenharia Agrícola e Ambiental, Campina Grande, v. 11, n. 1, p. 67-72. 2007.

SORIANO, B.M.A. Caracterização climática de Corumbá-MS. Boletim de Pesquisa, 1. Corumbá: EMBRAPA-CPAP, p.25. 1997.

TUBELIS, A.; NASCIMENTO, F.J.L. Meteorologia Descritiva: fundamentos e aplicações brasileiras. São Paulo: Nobel, 1992. 